\title{
Pulmonary metastasectomy in soft tissue sarcomas: a systematic review
}

\author{
Davor Stamenovic ${ }^{1}$, Peter Hohenberger ${ }^{2}$, Eric Roessner ${ }^{1}$ \\ ${ }^{1}$ Mainz University Thoracic Center, Thoracic Surgery and Pulmonary Medicine, Mainz, Germany; ${ }^{2}$ Division of Surgical Oncology \& Thoracic \\ Surgery, Mannheim University Medical Center, Mannheim, Germany \\ Contributions: (I) Conception and design: D Stamenovic; (II) Administrative support: D Stamenovic; (III) Provision of study materials or patients: \\ D Stamenovic; (IV) Collection and assembly of data: D Stamenovic; (V) Data analysis and interpretation: D Stamenovic, P Hohenberger; (VI) \\ Manuscript writing: All authors; (VII) Final approval of manuscript: All authors. \\ Correspondence to: Davor Stamenovic. Mainz University Thoracic Center, Thoracic Surgery and Pulmonary Medicine, Mainz, Germany. \\ Email: davor.stamenovic@unimedizin-mainz.de.
}

\begin{abstract}
Background: Soft tissue sarcoma (STS) tend to metastasis to the lungs. Pulmonary metastasectomy seems to be a common practice always when plausible. The objective of this article was to review systematically the results of a literature search on pulmonary metastasectomy for STSs published in the last ten years and to offer a brief overview about the current practice as well.

Methods: Eight retrospective studies published in the period 2010-2020, which included patients with pulmonary metastases and metastasectomy were selected. Indication for surgery, survival rate and factors influencing survival were the primary outcomes, while further interesting findings in the studies were also collected and evaluated.

Results: Cumulative 1,004 patients participated in these studies. The most common histological types were leiomyosarcoma, malignant fibrous histiocytoma $(\mathrm{MFH})$ and synovial sarcoma, being present together at $60 \%$ of the study population. Five-year survival was reported to be in the range from $20-58 \%$, better survival going along with a fewer (preferably one) metastases, longer disease free interval (DFI) and R0 resection in most of the cases.

Conclusions: Complete resection of the metastatic lesions seems to be the most effective treatment for long-term survival, or even achieving cure in selected patients. At selection of the patients amenable for surgery, a high probability of R0 resection, as well as a disease free period of at least 12 months should perhaps bear a higher specific value.
\end{abstract}

Keywords: Soft tissue sarcoma (STS); pulmonary metastasectomy; lung metastases

Submitted Apr 29, 2020. Accepted for publication May 11, 2020.

doi: $10.21037 /$ jtd-2019-pm-13

View this article at: http://dx.doi.org/10.21037/jtd-2019-pm-13

\section{Introduction}

Under the common nomenclature of soft tissue sarcoma (STS, ICD-10 C47-C49), heterogeneous groups of tumors are summarized which consist of more than 80 different histological entities $(1,2)$. The incidence of these tumors is approximately 6 per 100,000 individuals a year, representing $1 \%$ of all adult malignancies.

The adequate treatment of STSs is challenging not only regarding the strategy for the primary tumors, but also when they are at the metastatic stage (3).

Due to their histological differences, varieties in the pathogenesis as well as the genetic alterations, they present themselves as entities with extremely variable clinical behavior. Roughly, half of STS subtypes can be characterized by redundant chromosomal rearrangements and genetic alterations. The other half typically shows complex karyotypes and denomination of subtypes changes 
and overlaps over years, e.g., from malignant fibrous histiocytoma (MFH) to myxofibrosarcoma to not otherwise specified (NOS) to undifferentiated pleomorphic sarcoma (UPS) (4). This is partially the reason, along with their rarity, for the absence of high quality evidence based studies regarding their treatment, particularly concerning pulmonary metastases, as the main target organ in the advanced stage of the disease. Currently there is no evidence level better than IIIa (systematic review with homogeneity of case-control studies) (5), which comprised the studies up to 2010, and including the metastatic bone sarcomas in the analysis.

The objective of this review article was to review systematically the results of a literature search on pulmonary metastasectomy for STSs published in the last ten years, as well as to offer a brief overview about the current practice. We present the following with the PRISMA reporting checklist (available at: http://dx.doi.org/10.21037/jtd-2019pm-13).

\section{Methods}

First, we conducted a PubMed search of the available publications according to the PRISMA (6) recommendations. Eligibility criteria were:

(I) Publication in English;

(II) Original studies with at least 25 patients undergoing primary metastasectomy solely for STS with reported overall survival (OS);

(III) Date of publication after 01.01.2010.

Then a PubMed search was conducted with the following terms contained in the title/abstract: Sarcoma (AND) metastasectomy (AND) lung, as well as sarcoma (AND) metastasectomy (AND) pulmonary.

The results were evaluated according to the quality of summarizing in the abstracts and filtrated on the presence of the inclusion criteria. After the first filtration, the full article text was retrieved from the remaining publications and reevaluated for the inclusion into the systematic review. Furthermore, several corresponding authors of the selected articles have been contacted via email in order to provide some additional data or clarification.

Following data were extracted:

(I) Methodology of the study;

(II) Demographic characteristic of the study population: number, age and sex;

(III) Histological type of the STS;

(IV) OS presented whether as a median survival in months or percentage of the cohort reaching the five-year survival benchmark;

(V) Data on disease free survival (DFS) post metastasectomy;

(VI) Use of systemic anticancer chemotherapy;

(VII) Factors influencing survival according to the study;

(VIII) The number of patients with additional extrapulmonary metastases;

(IX) Pulmonary/mediastinal lymph node involvement;

(X) Presence of synchronous pulmonary metastases;

(XI) Time interval between the primary tumor treatment and the pulmonary metastasectomy;

(XII) The type of surgery (VATS or open), R0 resection rate, complications and mortality;

(XIII) The number of resected metastases;

(XIV) The number of patients with repeated surgery;

(XV) Reporting period;

(XVI) Median tumor size and range;

(XVII) Local recurrence rate;

(XVIII) Further interesting findings in particular studies.

\section{Studies and study details}

The initial search yielded 72 and 88 studies respectively. The first screening process retained 25 studies for detailed examinations whereas the rest of the studies were excluded according to the specified criteria. Furthermore, 17 studies were excluded because they did not provide detailed results on STS or those results were not possible to be separated from all reported results. Also, some studies reported inconclusive results or duplicate data were given.

After all, we retained 8 publications published from 01.01.2010 to present with in total 1,004 patients having undergone pulmonary metastasectomy for STS (7-14).

Distribution of the histologic subtypes the resections were performed for are given in Table 1 .

Among the selected studies, there were no randomized controlled trials. All the studies were retrospective cohort studies conducted from institutional databases. One study offered comparison between two groups of patients undergoing pulmonary metastasectomy either with or without neoadjuvant chemotherapy (13) and another one compared the outcomes among the surgical and nonsurgical groups (8), however none of them had prospective and protocol-based selection criteria.

While all of the studies analyzed heterogeneous group 
Table 1 Distribution of histologic subtypes in patients undergoing pulmonary metastasectomy for STS

\begin{tabular}{lcc}
\hline Diagnose & Number & Percent (\%) \\
\hline Leiomyosarcoma & 239 & 24 \\
Malignant fibrous histiocytoma & 199 & 20 \\
Synovial sarcoma & 164 & 16 \\
Liposarcoma & 62 & 6 \\
Fibrosarcoma (incl. Myxofibrosarcoma) & 86 & 5 \\
Undifferentiated pleomorphic sarcoma & 49 & 3 \\
Malignant peripheral nerve sheath tumor & 29 & 17 \\
Undetermined and others & 176 & 100 \\
Total & 1,004 & 5 \\
\hline
\end{tabular}

STS, soft tissue sarcoma.

of patients with various STS, one study focused solely on a single subtype-synovial sarcoma (11). The retrospective time frame of the 8 reports ranges from 1980-2016. The histological subtypes referred to in the single publications often are differently described today.

A few studies provided data on a cohort of STS patients from which a subgroup with pulmonary metastases and eventually with pulmonary metastasectomy were selected $(8,10,11,13)$. One study reported on a patient population with pulmonary metastases and subsequently with pulmonary metastasectomy (12), whereas all other authors described the patient group undergoing resection exclusively. Hence, the proportion of the patients developing pulmonary metastases, which was calculated from those four studies were $9 \%, 28 \%, 34 \%$ and $21 \%$ respectively, whereas the proportion of the population eventually undergoing lung metastasis resection was $5 \%$ ( $51 \%$ of those with pulmonary metastases), $8 \%(30 \%)$, $20 \%(58 \%)$ and $4 \%(21 \%)$ respectively. The proportion of the patients with pulmonary metastases undergoing metastasectomy in the remaining above-mentioned study (without the absolute number of STS patients) was $67 \%$.

The inclusion and exclusion criteria for pulmonary metastasectomy were not elaborated in all studies.

Nevala et al. (8) compared a surgical with a non-surgical group. From initially 1,580 patients with STS, only 145 (9\%) developed pulmonary metastases and of these, 130 patients were enrolled in the study. Out of these patients 74 (5\%) underwent surgery. Among 56 patients not amenable to surgery, there were 19 patients with an insufficient Eastern Cooperative Oncology Group (ECOG) score higher than 1, 15 patients with multiple pulmonary lesions (without further details), 15 patients with a too difficult anatomical location of pulmonary lesions, 4 patients denied surgery, while another two patients had a short disease free interval (DFI) and/or rapid progression before the scheduled surgery. Out of the non-operated group, 36 patients received palliative chemotherapy while another 20 patients had best supportive care. The authors had excluded all the patients with additional extrapulmonary metastases.

Schur et al. (10) report on 46 patients out of 108 metastatic patients (out of 552 patients with STS). Exclusion criteria for a surgical approach were pulmonary advanced disease $(\mathrm{n}=15)$, multiple intrapulmonary nodules $(n=34)$ additional extrapulmonary metastases $(n=55)$, poor performance status $(\mathrm{n}=3)$ and one refusal.

Lee et al. (11) used a rather simple algorithm; they attempted to perform surgery in all cases whenever complete resection seemed to be feasible, regardless of the size and number of nodules.

In the paper from Ohnstad et al. (13) 2,504 patients had initially been diagnosed with STS and 525 (21\%) developed pulmonary metastases in the course of the disease. However, $112(4 \%)$ underwent pulmonary metastasectomy, from whom only 93 managed to be included into the study. The exact exclusion criteria are not precisely reported except for excluding patients with synchronous pulmonary metastases $(\mathrm{n}=175)$ from surgery and omit from further analysis. In this study, as well as in a few others $(7,10,12)$, the presence of additional extrapulmonary metastases did not represent an exclusion criterion, nor it was the presence of local recurrence at the site of the primary tumor $(7,8,12,13)$. 
Chudgar et al. (12) included 9 (2\%) patients with both, local recurrence and additional extrapulmonary metastases to their series. Toussi et al. (9) and Predina et al. (7) do not report these data.

\section{Results}

In all studies but one (10), besides the calculation of OS given as a median in months, the stated purpose was to assess prognostic factors related to the estimated survival. The tabular overview of the studies along with the demographics and clinical results is given in Table 2, whereas the results from analyzing prognostic factors regarding survival after pulmonary metastasectomy are given in Table 3.

The most relevant findings out of the studies are summarized in the following sections. Predina et al. (7) did not find any difference in survival between the patients receiving postoperative chemotherapy $(n=31,65 \%$ of resected) and those without. Most of the patients underwent chemotherapy with doxorubicin/ifosfamide $(n=20)$, while a few received alternative protocols: protocol with vincristine, gemcitabine, docetaxel, carboplatin, and etoposide $(n=6)$ and undetermined $(\mathrm{n}=5)$. Median DFS after metastasectomy for patients receiving chemotherapy was 1.1 years, while it was 1.2 for patients not receiving any $(\mathrm{P}=0.88) 5$-year $\mathrm{OS}$ for the postoperative adjuvant chemotherapy group was 4.9 years compared to 5.3 years for patients receiving none ( $\mathrm{P}=0.41)$. Schur et al. (10) calculated 5-year survival rates for both, the diagnosis of the primary tumor as well as the first pulmonary metastasectomy as shown in Table 2. The survival rate after the first pulmonary metastasectomy was $32 \%$, in comparison to $62 \%$ after diagnosis of the primary tumor.

Chudgar et al. (12) found R1/R2 resection not to be a prognostic significant factor $(\mathrm{P}=0.2)$, whereas preoperative chemotherapy was accompanied with a significantly higher hazard ratio of $(1.04,1.77 ; \mathrm{P}=0.025)$.

Ohnstad et al. (13) had two subgroups in their cohort, either with neoadjuvant therapy $(n=52)$ or without $(n=41)$, but with no significant difference in median survival. Moreover, there was a subgroup of poor histological responders, i.e., radiological progression to chemotherapy $(\mathrm{n}=37)$ with detrimental prognosis (PFS $=16$ months) This reminds to the fact that even in the most promising chemotherapy combination of doxorubicin/ifosfamide the rate of partial remissions was $26 \%$ in a prospective phase III trial leaving almost three fourths of the patients with no decrease in tumor size preoperatively (15).
A summary of the surgical treatment of pulmonary metastases is presented in Table 4.

A clear preference towards open resections is obvious in all of the series, although in most of them the median number of resected metastases was only two with a range from 1 to 52.

Predina et al. (7) reported VATS resection in 13 (27\%) patients, all with less than three nodules on preoperative imaging. However, they also reported the mean number of nodules was 1.6 on computed tomography imaging before the pulmonary metastasectomy while the mean number of pathologically proven nodules was $2.3(\mathrm{P}=0.08)$. Seven patients underwent three or more resections, three of whom achieved long-term survival of 5, 8 and 12 years respectively. Nevala et al. (8) reported VATS in 18 (24\%) patients and Schur et al. in 3 (4\%). Out of 16 patients undergoing repeated metastasectomy, three patients underwent surgery 3 times and one patient 9 times. Lee et al. (11) performed VATS in 5 out of 50 resections, 5 of the patients undergoing metastasectomy for 3 times, 2 patients for 4 times, and one patient for 7 times, being still alive 11 years after the first metastasectomy. Chudgar et al. (12) reported 156 (29\%) resections with VATS, 127 of them being after 2004 and only 29 before that year. Eighty-one\% of the procedures were for resection of 1 or 2 pulmonary metastases.

\section{Discussion}

There is an ongoing debate whether pulmonary metastases of STS can contribute to long-term survival of the patients and whom and when to select for lung metastases resection (16). One of the best evidences can be concluded from the recent French METASARC study (17). The study included more than 1,600 patients with metastatic STS only and analyzed their long-term survival. It turned out, that patients who had undergone surgical treatment for metastatic disease had a clear survival benefit. The study data refer to patients with all sites of metastatic disease and did not provide a subgroup analysis. However, as patients with lung metastases accounted for $65 \%$ of the cohort, the data can be used as a clear argument in favor of resection of lung metastases.

This systematic review aimed to evaluate the recent publications on pulmonary metastasectomy for any STS to put more light on the debate. However, after analyzing the selected papers the question whether pulmonary metastases of STS should be resected, and if so under which circumstances, and when, can still hardly be answered. 


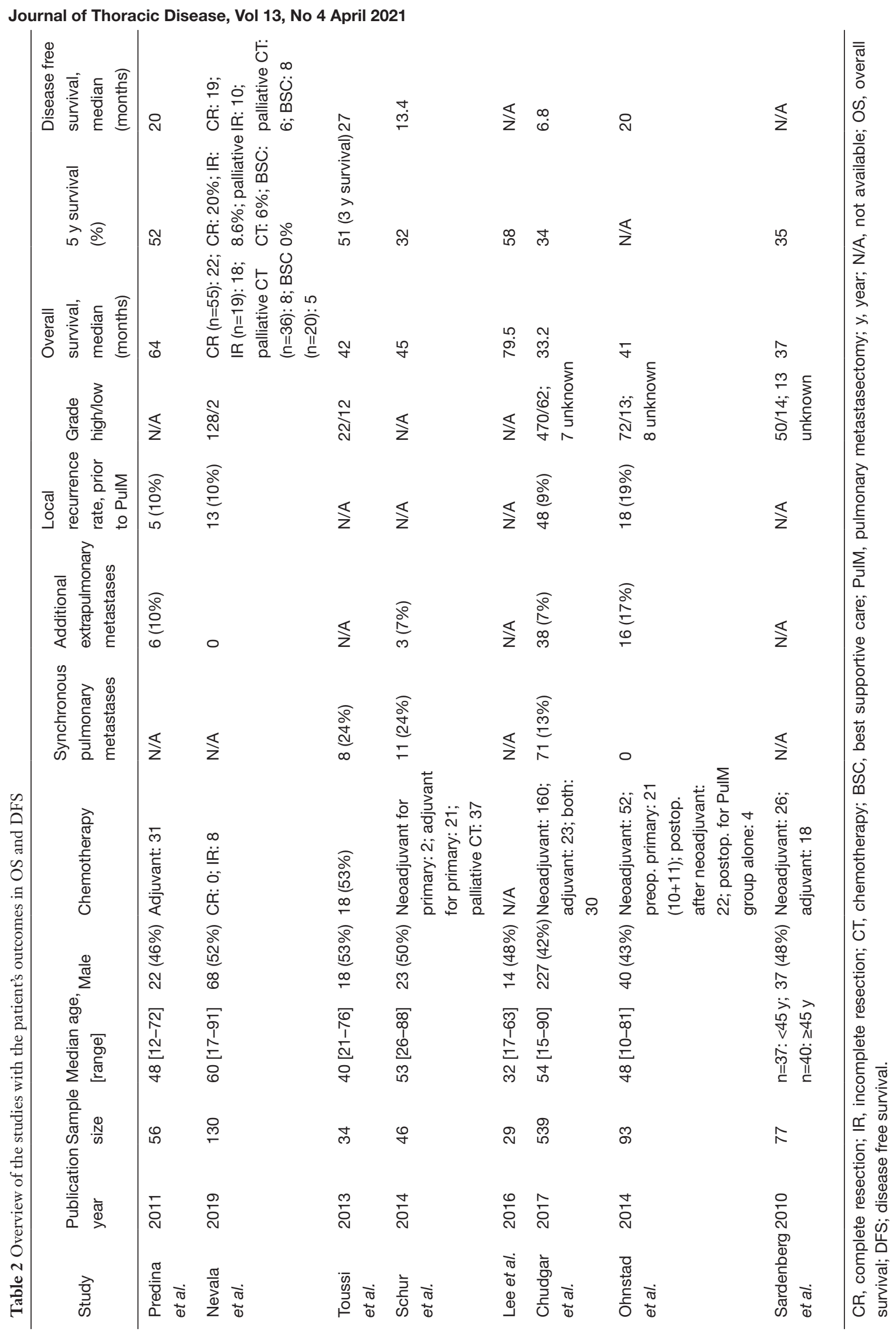


Table 3 Factors influencing survival

\begin{tabular}{|c|c|}
\hline Study & Factors influencing survival \\
\hline \multirow[t]{3}{*}{ Predina et al. } & 1. having $\leq 2 \mathrm{PL}$ \\
\hline & 2. having a largest $P L<2 \mathrm{~cm}$ \\
\hline & 3. having no extrapulmonary metastases \\
\hline \multirow[t]{3}{*}{ Nevala et al. } & 1. single PulM \\
\hline & 2. $\mathrm{DFI}>1.3$ years \\
\hline & 3. $\mathrm{R} 0$ resection \\
\hline \multirow[t]{3}{*}{ Toussi et al. } & 1. $\mathrm{R} 0$ resection \\
\hline & 2. DFI >18 months \\
\hline & 3. Unilateral PulM \\
\hline Schur et al. & N/A \\
\hline \multirow[t]{2}{*}{ Lee et al. } & 1. Unilateral PulM \\
\hline & 2. DFI $>12$ months \\
\hline \multirow[t]{5}{*}{ Chudgar et al. } & 1. Leiomyosarcoma subtype \\
\hline & 2. Primary tumor size $<10 \mathrm{~cm}$ \\
\hline & 3. DFI >12 months \\
\hline & 4. Solitary PulM \\
\hline & $\begin{array}{l}\text { 5. Absence of additional extra PulM/local } \\
\text { recurrence }\end{array}$ \\
\hline \multirow[t]{3}{*}{ Ohnstad et al. } & 1. Better histological response to CT (Huvos IV) \\
\hline & 2. Radiological response to $\mathrm{CT}$ \\
\hline & 3. DFI >6 months \\
\hline \multirow[t]{3}{*}{ Sardenberg et al. } & 1. Number of PulM resected $<4$ \\
\hline & 2. $\mathrm{DFI}>16$ months \\
\hline & 3. $\mathrm{R} 0$ resection \\
\hline
\end{tabular}

DFI, disease free interval; CT, chemotherapy; PulM, pulmonary metastases; N/A, not available.

The reviewed studies advocate surgery for pulmonary metastases of STS and no study reports on a control group. This makes it obvious that only a small percentage of highly selected patients initially presented with pulmonary metastases had the chance of having surgery, resulting in a publication bias. There are no data from prospective randomized trials as the only study intended (EORTC 62933. NCT00002764) had to be closed early for very slow accrual despite involving multiple international groups (18).

How those patients were selected precisely remains unrevealed in most cases. It still makes most sense to stick to Treasure et al.'s (5) last systematic review to Thomford et al.'s (19) historical study the inclusion criteria for surgery being:

(I) Primary cancer eradicated (controlled or amenable to control);

(II) Pulmonary metastases amenable for R0 resection;

(III) No additional extrapulmonary metastases; and

(IV) Acceptable lung function for the amount of resection.

It then becomes apparent, that the classical "rules" still had a major impact on the decision making process. Only a small percentage of the patients undergoing pulmonary metastasectomy were recruited from subgroups having an additional extrapulmonary metastases and/or local recurrence. And theses subgroups-if properly reported showed worse survival data. Another small percentage of the selected patients who could not be resected completely, which in most cases would probably have not been operated with a better preoperative judgment in selecting mostly those amenable to R0 resection.

Two papers offered an alternative group(s) of patients to be compared with. From 130 patients with pulmonary metastases included in the study of Nevala et al. (8), 74 underwent surgery and 56 did not. Importantly, the nonsurgery group included patients who were not suitable for surgery, because of performance status, too many metastases, or too short DFI. A few patients refused the surgery. Ohnstad et al. (13) compared two surgical groups, one receiving induction chemotherapy and other proceeding with immediate surgery. They found no difference between the groups.

Six studies evaluated the a prolonged DFI represents the most consistent prognostic factor. Two studies were setting a typically consented value of 12 months, whereas two studies used 16 months as a critical threshold. One study set it on 6 and another on 18 months. Earlier publications claimed that "more is better"- the longer the DFI better the prognosis typically one or two years, however the cutoff value was set to be different in various studies (20-22).

The number of resected metastases represents another consistent prognostic factor in the literature. Six out of eight papers deemed it significant and the message is the less the better. However, if we would look for an upper limit on the number to indicate resection, it would have been very flexible as it reached 30 metastases and more in at least three studies, two of them with highest reported R0 resection rate within our review.

While solitary lung metastasis in the lung should represent a clear indication for local treatment-which is 


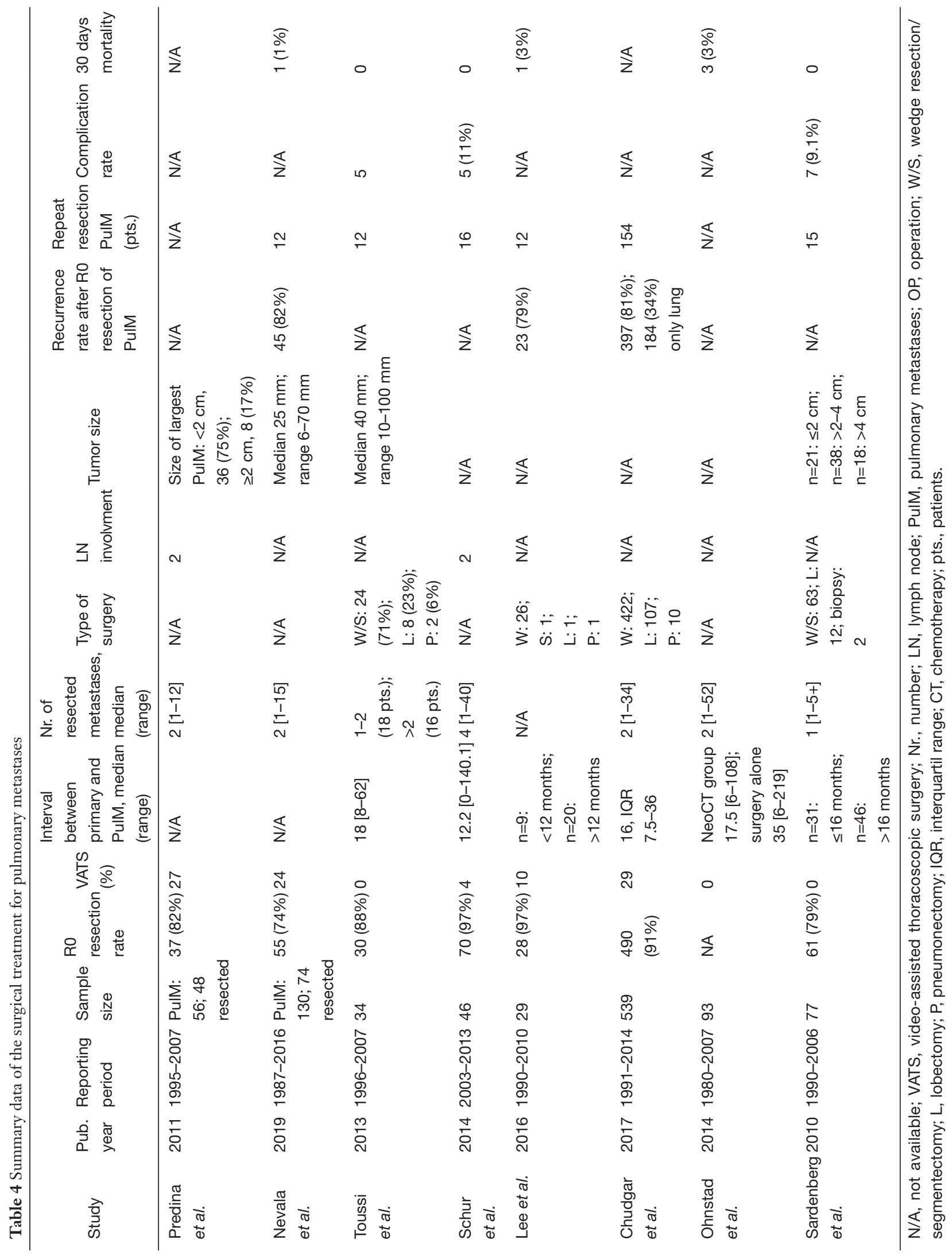


in many cases the surgical option, multiple disseminated metastases over both lungs are a clear for non-operative options, if the sarcoma subtype is sensitive to chemotherapy, like synovial sarcomas, or metastases of pleomorphic high grades sarcomas. However, the situation might be different for metastases of clear cell sarcomas, extraskeletal myxoid chondrosarcomas, epithelioid sarcomas, or alveolar soft part sarcomas-subtypes which are regarded to be non-sensitive to systemic chemotherapy and also do not provide an option for targeted therapy $(23,24)$. In such cases, surgical treatment might be an option and possibly those were the subtypes in the publications removing 30 metastases and more. Unfortunately, these facts are not commented in the reports.

Expected completeness of resection bears the attribute "condition sine qua non" when it comes to preoperative evaluation and decision for surgery, thus this condition is almost unequivocally present.

Predina et al. (7) found, however, no difference in OS $(\mathrm{P}=0.33)$ or DFS $(\mathrm{P}=0.99)$ regarding the positive margin after metastasectomy, which occurred in $18 \%$ of the cases. In case of OS, significance was reached after the expansion of the sample.

Chudgar et al. (12) also failed to demonstrate a significance to that matter, maybe because of the small proportion of R1 resections as well. Two other recent publications confirm this finding $(25,26)$.

Histology and grading did not appear to be of a huge importance in the reviewed studies. Chudgar et al. (12) were the only group finding a particular histology prognostically advantageous. Although leiomyosarcoma histology was found to be associated with longer OS (42 months) compared to other histology subgroups, fibrosarcoma histology was even better to that matter reaching the longest median OS (65.2 months); however, $42 \%$ of this subgroup had low-grade sarcomas as the group of fibrosarcomas is split in a huge number of prognostically very different subgroups making retrospective review difficult to interpret. Regarding DFS, there was a significant advantage for patients with low grade primary tumor $(\mathrm{P}=0.017)$, and subsequently these patients also showed better survival rates $(\mathrm{P}<0.001)$. The group of Toussi et al. (9) also report a similar trend $(\mathrm{P}=0.18)$ but statistical significance is not reached due to small sample size.

Other selected studies failed to find any significance regarding histological subtype of the sarcoma and, more important, its grading.

Grading of the sarcomas should be an important factor indeed, as it associates with tumor aggressiveness and tendency for early and widespread dissemination (27).

A few older studies supported the findings of Chudgar et al. (12) regarding positive impact of leiomyosarcoma histology on survival $(25,28,29)$. Other studies found contradictory results regarding $\mathrm{MFH}$ to be connected to a more advantageous course compared other subtypes (29-31). This disease entity as of today does no longer exist in the WHO classification, thus the results mentioned are hard to take into account in today's decision-making.

One recent study found synovial sarcoma histology being associated with disadvantageous prognosis (25), another study claimed the opposite (22), maybe due to the fact that synovial sarcoma belonged to the group of chemo-sensitive (doxorubicin/ifosfamide) sarcomas.

The idea of histology-driven treatment looks appealing (32). However, the decision for lung metastasis surgery is still a "second-line" consideration, except in only a few anthracycline (doxorubicin) resistant histological types (alveolar soft part sarcoma, solitary fibrous tumor, hemangiopericytoma, clear cell sarcoma and others). In fact, a recently conducted study evaluating neoadjuvant chemotherapy by comparing a standard drug regimen (doxorubicin/ifosfamide) versus a so-called tailored approach with histotype-specific chemotherapy demonstrated that standard chemotherapy is advantageous (33).

Recurrences and their resectability and/or operability have often been an issue in the past.

Nearly all of the selected publications reported remetastasectomy, but not necessarily a recurrence rate as well (Table 4). Although a few of the papers found a survival advantage in the subgroup of patients undergoing repeated resections $(10,14)$, others either did not (9) or did not analyze it $(8,11,12)$.

There is a belief and an explanation why the patients undergoing repeated resections may have a survival advantage. This phenomenon emerges probably due to selection of the patients with less aggressive tumor and a biological behavior of longer intervals between the onset and development of metastases. Many of these patients with favorable outcome fulfill the criteria of oligometastatic disease (34-36).

The majority of patients with a tumor recurrence deemed not to be suitable for surgery anymore, probably due to widespread dissemination or co-morbidity factors precluding further surgical treatment.

Systemic therapy (ST) was inconsistently used in the selected publications. While nearly all authors reported 
the application of ST for the primary tumor pre- or postoperatively, as well as for the metastatic tumor setting it was not hardly clear what were the indications for one group of patients to receive it and another not. Only one study compared two surgical arms, one of which receiving induction ST (with diverse protocols). The survival results were not significantly different (11).

Standard drug in metastatic STS is doxorubicin $\left(75 \mathrm{mg} / \mathrm{m}^{2}\right)$ as a single agent resulting in a response rate reported to be approximately $14 \%(15)$. When used in comparative trials versus eribulin or trabectedin, the response rate according to Response evaluation criteria in solid tumors (RECIST) $1.0 / 1.1$ ranged from $0 \%$ to $21 \%$. A combination of $75 \mathrm{mg} / \mathrm{m}^{2}$ of doxorubicin and $10-12 \mathrm{~g} / \mathrm{m}^{2}$ of ifosfamide yields a significantly higher response rate of $26 \%$ based on RECIST criteria (37) could be found. In both study arms $20 \%$ of the patients underwent surgery of their metastases, however, this did not transfer to an improved OS rate (15). It was concluded that the combination regimen should be favored in a neoadjuvant setting. Trabectedin and eribulin are newer anti-sarcoma drugs, the former being approved as a secondline therapy for leiomyosarcoma and liposarcoma, while the later was approved for therapy of liposarcoma only, however with significant adverse effects (grade III 63\% vs. $53 \%$, grade IV $26 \%$ vs. $20 \%$ and fatal $4 \%$ vs. $1 \%$ ) (38). In leiomyosarcomas, gemcitabine combined with docetaxel was considered to be of major value, however, a comparative study to single-agent doxorubicin showed that the combination added toxicity but not an outcome advantage (39).

Targeted therapy drugs emerge lately as valid agents in therapy of diverse malignant diseases. Regarding metastatic STS a few agents are under investigations. In the secondline and third-line setting pazopanib failed to show benefit in OS compared with a placebo group (40). However, due to better progression-free survival and despite low response rate and its adverse effects, the agent was approved for treatment of non-adipocytic STS. It must be borne in mind that surgery following pazopanib should be conducted after a at least two weeks interval as wound healing problems might occur due to interference with VEGF.

Response to ST, more particularly progression despite administration of ST, was a poor prognostic factor in regard to pulmonary metastasectomy (26). Such a scenario moves a surgical approach clearly towards being a palliative procedure-except in selected single cases. Actually, there is no sufficient data supporting the use of ST in either the neoadjuvant or adjuvant settings, after R0 resection of pulmonary metastases in STS.
Miscellaneous other factors like the size of the biggest metastasis (7) appear to be backed-up in the literature (41-43). Age above 50 (44), or maybe 55 years of age (45), might be more misleading, as sarcomas in adults typically are detected beyond the age of 60 . Radiological response on ST, in particular when correlating to histological response, was a prognostically significant factor in a highly selected patient group in one study (13).

This survey clearly depicts that the current level of evidence is low, based on exclusively retrospective, nonrandomized cohort reports. It suggests improved overall OS "due" to surgery. In our view, there might be an association but with many cofounders, particularly tumor biology. To start randomized trials is a must in today's times.

\section{Conclusions}

Pulmonary metastasectomy is widely accepted as a standard treatment approach for lung metastases from STS, and complete resection of the metastatic lesions should be the most effective treatment for long-term survival, or even achieving cure in selected patients.

There is consensus between the interdisciplinary expert groups that surgical resection of pulmonary metastases of STSs is encouraged and indicated, if (46):

(I) R0 resection of all pulmonary metastases is expected;

(II) There are no signs of local tumor recurrence at the site of the resected primary tumor that cannot be controlled locally;

(III) Prior pulmonary metastasectomy is no contraindication;

(IV) In metachronously resected lung metastases there is no indication for additive chemotherapy.

However, in case of the presence of extrapulmonary metastases, surgical removal of pulmonary metastases must be an interdisciplinary-based single case decision. Proven predictive factors for improved OS rates are: the time interval between the removal of primary tumors and the first detection of lung metastases of more than 12 months and $\mathrm{R} 0$ removal of the lung metastases. The resection for lung metastases with palliative intent is generally not recommended and should be restricted to patients suffering from significant symptoms induced by the metastases.

\section{Acknowledgments}

We would like to express our very great appreciation to Dr. 
Thomas Kindler and Dr. Frank Traub for their valuable and constructive suggestions during the planning and development of this research work.

Funding: None.

\section{Footnote}

Provenance and Peer Review: This article was commissioned by the Guest Editor (Khosro Hekmat) for the series "Pulmonary Metastases" published in Fournal of Thoracic Disease. The article has undergone external peer review.

Reporting Checklist: The authors have completed the PRISMA reporting checklist, available at http://dx.doi. org/10.21037/jtd-2019-pm-13

Data Sharing Statement: Available at http://dx.doi. org/10.21037/jtd-2019-pm-13

Conflicts of Interest: The authors have completed the ICMJE uniform disclosure form (available at: http://dx.doi. org/10.21037/jtd-2019-pm-13). The series "Pulmonary Metastases" was commissioned by the editorial office without any funding or sponsorship. The authors have no other conflicts of interest to declare.

Ethical Statement: The authors are accountable for all aspects of this work in ensuring that questions related to the accuracy or integrity of any part of this work are appropriately investigated and resolved.

Open Access Statement: This is an Open Access article distributed in accordance with the Creative Commons Attribution-NonCommercial-NoDerivs 4.0 International License (CC BY-NC-ND 4.0), which permits the noncommercial replication and distribution of the article with the strict proviso that no changes or edits are made and the original work is properly cited (including links to both the formal publication through the relevant DOI and the license). See: https://creativecommons.org/licenses/by-nc-nd/4.0/.

\section{References}

1. Brennan MF, Antonescu CR, Moraco N, et al. Lessons learned from the study of 10,000 patients with soft tissue sarcoma. Ann Surg 2014;260:416-21; discussion 421-2.

2. Jo VY, Fletcher CD. WHO classification of soft tissue tumours: an update based on the 2013 (4th) edition.
Pathology 2014;46:95-104.

3. Amankwah EK, Conley AP, Reed DR. Epidemiology and therapies for metastatic sarcoma. Clin Epidemiol 2013;5:147-62.

4. Schaefer IM, Cote GM, Hornick JL. Contemporary Sarcoma Diagnosis, Genetics, and Genomics. J Clin Oncol 2018;36:101-10.

5. Treasure T, Fiorentino F, Scarci M, et al. Pulmonary metastasectomy for sarcoma: a systematic review of reported outcomes in the context of Thames Cancer Registry data. BMJ Open 2012;2:e01736.

6. Liberati A, Altman DG, Tetzlaff J, et al. The PRISMA statement for reporting systematic reviews and metaanalyses of studies that evaluate health care interventions: explanation and elaboration. PLoS Med 2009;6:e1000100.

7. Predina JD, Puc MM, Bergey MR, et al. Improved survival after pulmonary metastasectomy for soft tissue sarcoma. J Thorac Oncol 2011;6:913-9.

8. Nevala R, Jaamaa S, Tukiainen E, et al. Long-term results of surgical resection of lung metastases from soft tissue sarcoma: A single center experience. J Surg Oncol 2019;120:168-75.

9. Toussi MS, Bagheri R, Dayani M, et al. Pulmonary metastasectomy and repeat metastasectomy for soft-tissue sarcoma. Asian Cardiovasc Thorac Ann 2013;21:437-42.

10. Schur S, Hoetzenecker K, Lamm W, et al. Pulmonary metastasectomy for soft tissue sarcoma--report from a dual institution experience at the Medical University of Vienna. Eur J Cancer 2014;50:2289-97.

11. Lee K, Kang MC, Lee HW, et al. Pulmonary Metastasectomy in Adult Patients with Synovial Sarcoma: A Single-Center Experience. Korean J Thorac Cardiovasc Surg 2016;49:451-5.

12. Chudgar NP, Brennan MF, Munhoz RR, et al. Pulmonary metastasectomy with therapeutic intent for soft-tissue sarcoma. J Thorac Cardiovasc Surg 2017;154:319-30.e1.

13. Ohnstad HO, Bruland OS, Taksdal I, et al. Response to preoperative chemotherapy in patients undergoing resection of pulmonary metastasis from soft tissue sarcoma - a predictor of outcome? Acta Oncol 2014;53:1180-7.

14. Sardenberg RA, Figueiredo LP, Haddad FJ, et al. Pulmonary metastasectomy from soft tissue sarcomas. Clinics 2010;65:871-6.

15. Judson I, Verweij J, Gelderblom H, et al. Doxorubicin alone versus intensified doxorubicin plus ifosfamide for first-line treatment of advanced or metastatic soft-tissue sarcoma: a randomised controlled phase 3 trial. Lancet Oncol 2014;15:415-23. 
16. van Geel AN, Pastorino U, Jauch KW, et al. Surgical treatment of lung metastases: The European Organization for Research and Treatment of Cancer-Soft Tissue and Bone Sarcoma Group study of 255 patients. Cancer 1996;77:675-82.

17. Savina M, Le Cesne A, Blay JY, et al. Patterns of care and outcomes of patients with METAstatic soft tissue SARComa in a real-life setting: the METASARC observational study. BMC Med 2017;15:78.

18. van Geel AN, Rm van Der Sijp J, Schmitz PI. Which Soft Tissue Sarcoma Patients with Lung Metastases Should not Undergo Pulmonary Resection? Sarcoma 2002;6:57-60.

19. Thomford NR, Woolner LB, Clagett OT. The surgical treatment of metastatic tumors in the lungs. J Thorac Cardiovasc Surg 1965;49:357-63.

20. Pastorino U, Buyse M, Friedel G, et al. Long-term results of lung metastasectomy: prognostic analyses based on 5206 cases. J Thorac Cardiovasc Surg 1997;113:37-49.

21. Smith R, Pak Y, Kraybill W, et al. Factors associated with actual long-term survival following soft tissue sarcoma pulmonary metastasectomy. Eur J Surg Oncol 2009;35:356-61.

22. Cariboni U, De Sanctis R, Giaretta M, et al. Survival Outcome and Prognostic Factors After Pulmonary Metastasectomy in Sarcoma Patients: A 18-Year Experience at a Single High-volume Referral Center. Am J Clin Oncol 2019;42:6-11.

23. Schuetze SM, Patel S. Should patients with high-risk soft tissue sarcoma receive adjuvant chemotherapy? Oncologist 2009;14:1003-12.

24. Demetri GD, Baker LH, Beech D, et al. Soft tissue sarcoma clinical practice guidelines in oncology. J Natl Compr Canc Netw 2005;3:158-94.

25. Lin AY, Kotova S, Yanagawa J, et al. Risk stratification of patients undergoing pulmonary metastasectomy for soft tissue and bone sarcomas. J Thorac Cardiovasc Surg 2015;149:85-92.

26. Stephens EH, Blackmon SH, Correa AM, et al. Progression after chemotherapy is a novel predictor of poor outcomes after pulmonary metastasectomy in sarcoma patients. J Am Coll Surg 2011;212:821-6.

27. Kang S, Kim HS, Kim S, et al. Post-metastasis survival in extremity soft tissue sarcoma: a recursive partitioning analysis of prognostic factors. Eur J Cancer 2014;50:1649-56.

28. Burt BM, Ocejo S, Mery CM, et al. Repeated and aggressive pulmonary resections for leiomyosarcoma metastases extends survival. Ann Thorac Surg
2011;92:1202-7.

29. Blackmon SH, Shah N, Roth JA, et al. Resection of pulmonary and extrapulmonary sarcomatous metastases is associated with long-term survival. Ann Thorac Surg 2009;88:877-84; discussion 884-5.

30. Choong PF, Pritchard DJ, Rock MG, et al. Survival after pulmonary metastasectomy in soft tissue sarcoma. Prognostic factors in 214 patients. Acta Orthop Scand 1995;66:561-8.

31. Casson AG, Putnam JB, Natarajan G, et al. Five-year survival after pulmonary metastasectomy for adult soft tissue sarcoma. Cancer 1992;69:662-8.

32. Singhi EK, Moore DC, Muslimani A. Metastatic Soft Tissue Sarcomas: A Review Of Treatment and New Pharmacotherapies. P T 2018;43:410-29.

33. Gronchi A, Ferrari S, Quagliuolo V, et al. Histotypetailored neoadjuvant chemotherapy versus standard chemotherapy in patients with high-risk soft-tissue sarcomas (ISG-STS 1001): an international, open-label, randomised, controlled, phase 3, multicentre trial. Lancet Oncol 2017;18:812-22.

34. Kim S, Ott HC, Wright CD, et al. Pulmonary resection of metastatic sarcoma: prognostic factors associated with improved outcomes. Ann Thorac Surg 2011;92:1780-6; discussion 1786-7.

35. Hellman S, Weichselbaum RR. Oligometastases. J Clin Oncol 1995;13:8-10.

36. Weichselbaum RR, Hellman S. Oligometastases revisited. Nat Rev Clin Oncol 2011;8:378-82.

37. Ratan R, Patel SR. Chemotherapy for soft tissue sarcoma. Cancer 2016;122:2952-60.

38. Schöffski P, Maki RG, Italiano A, et al. Randomized, open-label, multicenter, phase III study of eribulin versus dacarbazine in patients (pts) with leiomyosarcoma (LMS) and adipocytic sarcoma (ADI). J Clin Oncol 2015;33:LBA10502.

39. Seddon B, Strauss SJ, Whelan J, et al. Gemcitabine and docetaxel versus doxorubicin as first-line treatment in previously untreated advanced unresectable or metastatic soft-tissue sarcomas (GeDDiS): a randomised controlled phase 3 trial. Lancet Oncol 2017;18:1397-410.

40. van der Graaf WTA, Blay JY, Chawla SP, et al. Pazopanib for metastatic soft-tissue sarcoma (PALETTE): a randomised, double-blind, placebo-controlled phase 3 trial. Lancet 2012;379:1879-86.

41. Yamamoto Y, Kanzaki R, Kanou T, et al. Longterm outcomes and prognostic factors of pulmonary metastasectomy for osteosarcoma and soft tissue sarcoma. 
Int J Clin Oncol 2019;24:863-70.

42. Luzzi L, Marulli G, Solli P, et al. Long-Term Results and Prognostic Factors of Pulmonary Metastasectomy in Patients with Metastatic Transitional Cell Carcinoma. Thorac Cardiovasc Surg 2017;65:567-71.

43. Welter S, Grabellus F, Bauer S, et al. Growth patterns of lung metastases from sarcomas. Virchows Arch 2011;459:213-9.

44. Billingsley KG, Burt ME, Jara E, et al. Pulmonary metastases from soft tissue sarcoma: analysis of patterns

Cite this article as: Stamenovic D, Hohenberger P, Roessner E. Pulmonary metastasectomy in soft tissue sarcomas: a systematic review. J Thorac Dis 2021;13(4):2649-2660. doi: 10.21037/jtd2019-pm-13 of diseases and postmetastasis survival. Ann. Surg. 1999;229:602-10; discussion 610-2.

45. Welter S, Grabellus F, Bauer S, et al. Growth patterns of lung metastases from sarcoma: prognostic and surgical implications from histology. Interact Cardiovasc Thorac Surg 2012;15:612-7.

46. Jochen Schütte SB, Thomas Brodowicz, Viktor Grünwald, et al. Weichgewebssarkome (maligne Weichgewebstumoren) des Erwachsenen. Onkopedia April, 2019. 